\title{
LAS 3R, EL PATRIMONIO Y EL LUGAR*
}

Artículo de Investigación - Recibido: 5 de diciembre de 2014. Aceptado: 12 de febrero de 2015

\author{
PhD. Juan Gabriel Ocampo Hurtado*
}

Universidad Nacional de Colombia, Sede Manizales - jgocampoh@unal.edu.co

\section{Para citar este artículo / to reference this article:}

Ocampo, J. (2015). Las 3R, el patrimonio y el lugar. Módulo Arquitectura CUC, Vol.14 N² 11-22. Doi:

\section{Resumen}

Reducir, reutilizar y reciclar, componentes de la regla de las 3R propuesta en la cumbre del G8 de 2004 como estrategia de desarrollo social, se plantean como diferentes niveles de intervención al patrimonio arquitectónico donde la condición fundamental es la protección al sentido de lugar.

\section{Palabras clave:}

Reducción, reutilización, reciclaje, patrimonio arquitectónico, lugar.

"Artículo producto de la investigación desarrollada en el marco de la línea de investigación: La relación entre la poética, la arquitectura y la enseñanza a partir del enfoque fenomenológico.

" Arquitecto, Universidad Nacional de Colombia, 1992; magister en multimedia educativa, Universidad de Barcelona, España, 2003 y Doctorado en Arquitectura, Diseño y Urbanismo, Universidad Autónoma del Estado de Morelos, Cuernavaca, México, 2009. Profesor asociado de planta y en dedicación exclusiva de la Universidad Nacional de Colombia ha sido Director de la Oficina de Ordenamiento y Desarrollo Físico de la Sede universitaria. Director del 


\section{THE 3R, HERITAGE AND PLACE}

\section{Abstract}

Reduce, reuse and recycle components of the 3R rule proposed in the 2004 G8 summit as a strategy of social development, is presented as different levels of intervention architectural heritage where the fundamental condition is to protect the sense of place.

\section{Keywords:}

Reduction, reuse, recycling, architectural heritage, place. 


\section{Las 3R y el patrimonio arquitectónico}

Si bien el reciclaje ha sido útil a lo largo de la historia de la humanidad, en el presente ha tomado un carácter prioritario ya que la confluencia de factores como la enorme producción de residuos y desechos, el alto costo de producción y el abuso a la biomasa, ha llevado a problemas globales de tipo ambiental y económico. La definición procedente de la Académica Española de la Lengua implica la preparación del material para que este pueda ser utilizado. En otras palabras se requiere el uso de energía y recursos para volver a hace útil dicho material. Evidentemente, esto ocurre independientemente de la forma o escala que tenga dicho material.

Gary Anderson experto en ordenación y planificación territorial ganó en 1970, cuando era estudiante de arquitectura el concurso para el diseño del símbolo universal del reciclaje. Fue tal su impacto y el de la tríada conformada por las palabras: reducir, reutilizar y reciclar, que en 2004 el Primer Ministro japonés, Koizumi Junichiro presentó en la cumbre del G8 la idea de 3R para definir al reciclaje como parámetro fundamental del desarrollo social.

Si bien, la regla de las tres erres tiene una relación directa con los productos de consumo masivo, vale la pena partir de estos conceptos para elaborar una interpretación a la relación entre reciclaje y patrimonio arquitectónico. De forma sinérgica a lo anterior, Reus afirma:
Frente a la "puesta en valor" del patrimonio en la que el proceso de rehabilitación se entiende como una "recuperación" de espacios, y que en la mayor parte de los casos no caben los ciudadanos sino los consumidores y usuarios (museos, hoteles, tiendas, etc.) el reciclaje patrimonial entiende el edificio como un impulso para los ecosistemas urbanos. El inmueble no es un elemento pasivo que se habilita, sino que se transforma aprovechando sus potencialidades y su carga significativa como estimulante social. (Reus, 2012).

El planteamiento de Reus es totalmente coherente con lo propuesto por Junichiro en la Cumbre del G8. El estimulante social propuesto reactiva el ecosistema urbano que transforma la experiencia de las comunidades humanas relacionadas con el patrimonio arquitectónico reciclado.

\section{La reducción y el residuo}

La organización GreenPeace (2014), recomienda tomar acciones para reducir la producción de objetos susceptibles de convertirse en residuos. Ello en términos generales implica el rediseño con el propósito de dar un manejo racional a los elementos utilizados en la fabricación o vida del producto. En el plano arquitectónico ello implicaría incluir, en todas las etapas del ciclo de vida del proyecto, el concepto de reducción como determinante económico y ambiental. 
En la fase inicial del proyecto arquitectónico o planeación se toman decisiones que afectan al entorno físico, al contexto cultural y en general al futuro del sitio que será intervenido. Es en esta etapa donde la reflexión al concepto de reducción en relación con el proyecto puede prevenir que los materiales a usar o el proyecto mismo, se conviertan en residuos. Es el momento idóneo para pensar no solo en el proyecto, sino en las circunstancias futuras que pueden afectar al sentido de la arquitectura como hecho cultural inserto en el entorno urbano. Díaz plantea:

"La arquitectura, entendida en su dimensión de hecho construido, desarrolla su potencial en la confinación de esos espacios donde los distintos usos tienen un lugar y resguarda su privacidad. En ese sentido y en su dimensión más primaria, una casa sería el lugar inicial de resguardo de la intimidad y sus subdivisiones internas la evidencia de las privacidades individuales que cobija." (Díaz, 2011).

Díaz da trascendencia al hecho construido. No solo existirán los elementos constitutivos de la obra, sino que en los espacios confinados se consolidará el lugar de resguardo y de la intimidad. Es decir, el lugar con la definición de identidad mas íntima.

La etapa de diseño alberga decisiones que afectan el futuro de la construcción y administración del proyecto. Un mate- rial mal elegido o un mal diseño puede acortar la vida del proyecto hasta la eventualidad de llevarlo a transformarse en residuo como resultado de la degradación física de sus componentes. Rosas afirma:

"De este modo, el proyecto de una nueva edificación empieza a ser entendido, más allá de su condición objetual, particular e individual, como un espacio de mediación, interacción, transición y estratificación del entorno en que el hombre vive y se manifiesta. En efecto, el territorio es parte co-sustancial de la arquitectura, forma parte de ella, la constituye y también, la materializa." (Rosas, 2007).

Rosas plantea la existencia futura del habitante como su interacción con el entorno de tal forma que supera la condición objetual del proyecto arquitectónico para elevarlo a la condición de lugar.

Durante el proceso de construcción la generación de residuos es usual ya que el material debe ser transformado para integrarse a la obra. La administración de la construcción definirá el efectivo uso de los materiales y por consiguiente el volumen de residuos resultante. En esta fase la relación entre recursos financieros, tiempo, tecnología y proveedores se verán como determinantes del éxito económico del proyecto en relación con la generación y desecho de residuos. 
Cuando el edificio se encuentra en su vida útil, la aparición de residuos se da por el tipo de funcionamiento y mantenimiento que este tenga. Es por ello que la administración del mismo edificio se hace necesario para efectos de proteger la integridad del conjunto constructivo. A modo de ejemplo, las medidas preventivas como la inmunización a elementos de madera puede prolongar su vida útil.

Con el paso del tiempo, hay edificios que pierden sus cualidades funcionales e incluso estéticas. Algunos de ellos pueden tener condiciones patrimoniales importantes de acuerdo con sus características culturales. Pero hay otros edificios que no representan ningún valor histórico que afecte al sentido de lugar y que por su alto deterioro se hayan convertido en desecho urbano. Al llegar a este estado, ni el conjunto ni sus materiales representan utilidad o la posibilidad de ser reciclados. Es cuando el edificio se ha convertido en residuo y procede la demolición y retiro de escombros.

\section{La reutilización}

Lecitra (2010) afirma que la reutilización "esta íntimamente relacionada con la prevención en la producción de residuos" y añade que "la adopción de medidas se centra principalmente en la reutilización de envases. Por su parte, la Real Académica de la Lengua Española define reutilizar como "utilizar algo, bien con la función que desempeñaba anteriormente o con otros fines.

Al tratar de entender estas dos ideas a partir del patrimonio arquitectónico, se plantea a la arquitectura como contenedor. ¿Pero contenedor de qué? Se podría responder a esta pregunta a partir de dos referencias al mundo clásico. La primera de ellas es una interpretación de Derrida al significado de Khôra propuesto por Platón:

\begin{abstract}
"Ya sea que conciernan al nombre mismo de Khôra ("lugar", "sitio", "emplazamiento", "región", "comarca") o a eso que la tradición llama figuras -comparaciones, imágenes, metáforas- propuestas por Timeo mismo ("madre", "nodriza", "receptáculo", "porta-impronta"), las traducciones quedan atrapadas en las redes de la interpretación" (Derrida, Pp5).
\end{abstract}

En Khôra el lugar existe para ser entendido como la conjunción de fondo y forma ya que según Platón, en el Timeo, no existe uno sin el otro. Khôra lleva al entendimiento de la concepción de arquitectura como lugar.

El segundo referente, discípulo de Platón, es Aristóteles quien en su Física, habla del lugar, del espacio y del vacío. Aristóteles afirma: 
"El lugar parece ser semejante a un recipiente, el cual es un lugar transportable, pero el recipiente no es una parte de su contenido. Así pues, en cuanto es separable de la cosa, no es la forma, y en cuanto la contiene, es distinto de la material". (Pp 118).

Mientras que en el Khôra de Platón el lugar incluye al continente y al contenido, en Aristóteles el espacio contiene a la forma. Es importante señalar que cuando Derrida interpreta a Khôra, hace referencia al Timeo como uno de los diálogos de Platón donde profundiza en la forma de llegar al conocimiento y para ello menciona la vía de la enseñanza razonada en conjunción con las ideas procedentes de la experiencia. Ello significa, a la postre, que Khôra incluye al contenido de fondo, procedente de la teoría y al de la forma física que es donde se vive la experiencia. Por otro lado, la definición de Aristóteles proviene de la naturaleza del texto, la física. Pero ambos coinciden en la importancia de la idea de contenedor que alberga el contenido. Felsenhardt se acerca a Khôra de la siguiente forma:

"Y digo país, porque "país", "pago", "paisaje", tienen una raíz común y el cambio de paisaje implica también un cambio en el país y en su carácter. Las nuevas viñas han contribuido a una modificación profunda, que va más allá de su propia coherencia vitivinícola; conlleva cambios en las costumbres rurales, en las labores del campo, en su renovada imagen y en su realidad tecnológica." (Felsenhardt, 2003)
En Felsenhardt el cambio del paisaje, además de implicar el aspecto físico, implica modificaciones en el carácter y en las costumbres. Son modificaciones que se dan dentro de la complejidad, ya que cambios en la forma pueden llevar a transformaciones culturales.

Si se acepta, en términos teóricos, la idea de arquitectura como contenedor, surge entonces la pregunta ¿cuál es el contenido? Si nos basamos en Platón, la arquitectura contiene fondo y forma que toma el significado de lugar al entrar en sinergia con la identidad cultural del sitio. Ello establece una fuerte relación entre Khôra y el patrimonio arquitectónico. Ya que el contenido formal de la arquitectura proviene de la disposición de sus materiales mientras que su contenido de fondo se refiere al sentido de su existencia desde el punto de vista epistémico, la conjunción entre estos dos tipos de contenido es lo que define el valor patrimonial de una obra de arquitectura. Asselot se refiere al paisaje de forma similar a Khôra de la siguiente forma:

"La definición de la palabra "paisaje" involucra al objeto de la representación y la representación en sí misma." (Asselot, 2003)

Felsenhardt y Asselot coinciden con la interpretación de Derrida frente a Khôra. El paisaje, el lugar, o la arquitectura, involucran en el sentido de su existencia al fondo y a la forma. 
Ahora, si se retoma la concepción de reutilización planteada inicialmente y si, como ya se dijo, fondo y forma es lo que esta contenido por la arquitectura, entonces la reutilización de la arquitectura significa su uso, bien con la función que tuvo en el pasado, o con otros fines. Esto no implica, necesariamente, la transformación de la obra de arquitectura. Su contenido de fondo y de forma pueden permanecer mientras su uso es replanteado. Ahora, si la obra es un bien de interés cultural, el contenido de fondo cobra relevancia ya que su significado determina las condiciones del lugar que en su entorno y contexto existen. En este caso la adopción de medidas debe ser cuidadosa de no afectar negativamente esa condición de lugar de tal forma que se proteja el sentido de identidad que implica dicho bien patrimonial. Crispiani plantea el tema desde la continuidad de la siguiente forma:

"Por otra parte, continuidad implica también movimiento y cambio dentro de una cierta pauta de orden inamovible. El principio de la buena forma es, en tal sentido, un principio fluido, que no intenta una unidad formal explícita y claramente reconocible en los objetos a los cuales se aplica, pero que implica una cierta coherencia entre ellos, coherencia que es tanto visible como comprensible." (Crispiani, 2001)

El aporte de Crispiani entrega mediante la coherencia en la continuidad se aplica perfectamente al estudio del patrimonio a partir de las 3R. La continuidad con el sentido de identidad entre el patrimonio arquitectónico y la comunidad que la creó y para la cual existe. Identidad que define al sentido de la existencia del lugar.

\section{El reciclaje}

Según la Real Academia Española de la Lengua reciclar significa "someter un material usado a un proceso para que se pueda volver a utilizar".

Esta definición implica que lo que fue usado en un momento entró en desuso y debido a circunstancias de cualquier tipo no se puede utilizar. Por ello se requiere un proceso que de viabilidad funcional a lo que se quiere reciclar. Martínez afirma:

\begin{abstract}
Denominar "reciclaje" a un proyecto de arquitectura indica, según se entiende en la definición que podemos encontrar, la voluntad de modificar las posibilidades de funcionamiento de un edificio en una nueva realidad; y pretende, de este modo, buscar nuevos usos en él, pues el anterior había quedado obsoleto o amortizado. (Martínez, 2012).
\end{abstract}

Si partimos de las palabras de Martínez, habría que analizar el estado de desuso en el que se encuentra el contenido de fondo y forma de la obra. Es posible que el contenido de fondo halla entrado en desuso por pérdida de identidad de la comunidad. De igual forma, el deterioro físico puede haber llevado la obra a un estado que implique 
alta vulnerabilidad física. Evidentemente, si el edificio es un Bien de Interés Cultural y ante la concurrencia del deterioro de fondo y de forma, la vulnerabilidad no solo será física sino cultural. De hecho en este punto no se considera la vulnerabilidad funcional, porque en este nivel, el edificio ya no es funcional.

Procede entonces la decisión sobre el reciclaje como proceso que dé nuevo uso al edificio o la demolición y renovación del sitio. Cualquiera de estas opciones modifican las condiciones de lugar como concepto de identidad. El reciclaje de la forma mediante el procesamiento de materiales, reposición, reemplazo o complemento estructural o funcional debe responder a las condiciones históricas del entorno y del contexto. La conciencia del pasado como procedencia del sentido de identidad, pero conciencia sobre el presente como circunstancia existencial dinámica. Como afirma Tejeda:

"Cualquier reflexión acerca del diseño patrimonial chileno pasa previamente por afirmar, negar o poner en duda nuestra historia republicana. Podemos suponer que, al fundarse la República, el país se cerró sobre sí mismo configurándose como un contexto y que en ese contexto empezaron a operar unos códigos específicos: sólo si aceptamos este supuesto, si de buen grado creemos que dentro de Chile se ha ido acumulando desde principios del siglo XIX un patrimonio visual, comunicacional y morfológico con leyes e identidad propias, tiene sentido hablar del tema.". (Tejeda, 2001)
Las palabras de Tejeda reivindican la identidad como factor existente en el patrimonio cultural. Dicho valor es fundamental ante el reto del reciclaje arquitectónico.

Nuevos materiales y tecnologías se incorporan al contenido formal durante la labor de reciclaje arquitectónico. Pero ¿como atender al reciclaje del contenido de fondo cuando se trata del patrimonio arquitectónico con valores culturales preponderantes? La respuesta se encuentra en la reivindicación al sentido de lugar.

\section{El lugar}

Este concepto ha formado parte de múltiples investigaciones a lo largo de la historia de la humanidad Ilegando a áreas del conocimiento como la filosofía o la antropología. Dada la relación establecida entre reciclaje, patrimonio y lugar, es importante profundizar en este concepto y para ello se propone la referencia a dos planteamientos: La cruz de Khôra, propuesta por Platón y el no lugar de Marc Augé.

En la cruz de Khôra el eje horizontal corresponde al "estar" y el eje vertical al "ser" de tal modo que un individuo puede "estar" en algún sitio, pero también "es". Pero, ¿como "es"? y ¿como "está"? determinan la condición existencial del habitante. Al confluir "estar" y "ser" en 
el centro de la cruz, hay relación directa entre ambas condiciones lo cual lleva a la conciencia del "ser" en el "estar". En otras palabras, el individuo es en el sitio de una forma consciente sobre lo que implica su existencia e historia individual en un sitio que alberga condiciones históricas y sociales de una comunidad. Dicha relación entre lo individual y lo general establece las condiciones de identidad entre el patrimonio arquitectónico y el habitante. La confluencia entre "ser" y "estar" se refiere a la arquitectura como lugar. Aravena se refiere a las fuerzas que determinan la arquitectura de la siguiente forma:

"Pero a pesar del peso evidente con que la arquitectura debe lidiar, en la práctica la gravedad aparece como una fuerza débil, equivalente y comparable al magnetismo. En arquitectura coexisten otras fuerzas aún mayores, a las que solo cabe aceptar. Muchas de ellas se originan en los hábitos y en la implacable vida cotidiana: la búsqueda del camino más corto que ofrece un atajo en el campo abierto, el deseo por una habitación que reciba la buena luz de la mañana y que ofrezca penumbra al anochecer, el intento por reducir los reflejos al interior del espacio de un edificio de oficinas acristalado -quizás demasiado moderno-donde la proliferación de cortinas pareciera proteger nuestros primitivos ojos." (Aravena, 2012)

Aravena hace énfasis en la conciencia de habitar, señala el camino fenomenoló- gico de la percepción de la arquitectura como lugar superando la sobre modernidad planteada por Augé (1996)y reivindicando la experiencia profunda de la arquitectura señalada por Saldarriaga (2002).

Con respecto al no lugar, Marc Augé hace desde la antropología un aporte a la relación entre el concepto de lugar y el patrimonio arquitectónico de la siguiente forma:

\section{"Si un lugar puede definirse como lugar de identidad, relacional e his- tórico, un espacio que no puede definirse como espacio de identidad ni como relacional ni como histórico, definirá un no lugar. La hipótesis aqui defendida es que la sobre moder- nidad es productora de no lugares, es decir, de espacios que no son en sí lugares antropológicos y que contrariamente a la modernidad bau- deleriana, no integran los lugares antiguos". (Augé, 1996)}

Si se toman la palabras de Augé para tratar de entender a la arquitectura como lugar, entonces la arquitectura tendría que tener sentido de identidad, tener relación sinérgica con los factores de entorno y contexto en los que existe y tener coherencia con el proceso histórico del sitio. Estas condiciones sirven para valorar el patrimonio arquitectónico. Holmes lo lleva a la práctica de la siguiente forma: 
"El encargo, la remodelación de la actual tienda Unicef, consistió básicamente en mejorar la utilización del lugar y aumentar la capacidad de almacenaje y exposición de productos. El proyecto se centró en 'limpiar' el lugar y en generar una lectura abstracta a partir del espacio despejado. Para ello se eliminaron muebles, cielos, tabiques, pavimento, iluminación, revestimiento de los muros. Sólo se conservaron los elementos que conformaban el espacio básico: un baño, dos pilares, una viga, los muros laterales, el vano frontal y una ventana en el muro del fondo. (Holmes, 2001).

La propuesta práctica de Holmes es coherente y respetuosa del sentido de reciclaje y de la obra ya que lleva el proyecto al mundo de las ideas donde lo abstracto de la concepción de lugar es analizado y a partir de allí se busca "mejorar la utilización del lugar". Buscó lo esencial de la obra y a partir de allí consolidó la respuesta a la actualización requerida.

\section{La investigación}

Estos planteamientos forman parte de la investigación "Lógica y Arquitectura" llevada a cabo en el grupo de trabajo académico de Medios de Expresión y Comunicación perteneciente a la Universidad Nacional de Colombia, Sede Manizales. En dicha investigación se estudiaron en profundidad temas como la percepción en la arquitectura, el concepto de lugar y la fenomenología en relación con temas del patrimonio arquitectónico como el de las 3R.

\section{Conclusiones}

- La iniciativa de la regla de las 3R presentada en la cumbre del G8 de 2004 tenía por objetivo central el desarrollo social en el mundo. Al reflexionar sobre la aplicación de la reducción, la reutilización y el reciclaje en el patrimonio arquitectónico surge la pregunta sobre el sentido de la intervención. Este sentido esta dado por el objetivo que pretendía el G8, el desarrollo social. Como se sabe, lo social es aquello relativo a la sociedad como conjunto de individuos que comparten una misma cultura y que interactúan para conformar una comunidad. Cuando un individuo se identifica como parte de una comunidad se da la identidad como relación entre los habitantes y el sitio que habitan. Se configura así el lugar. La regla de las 3R es por tanto compatible con el sentido del patrimonio arquitectónico.

- La reducción como principio que afecta todas las etapas de la vida del proyecto, se refiere al manejo económico del mismo. Ya sea en la planeación y diseño, o en la cons- 
trucción y en vida cotidiana de la obra, la conciencia sobre la reducción a la producción de residuos y la prevención a la transformación de partes del proyecto en residuo es una actitud de responsabilidad social, ambiental y financiera que afecta directamente a la arquitectura. El riesgo que muchas obras tienen debido al deterioro de los materiales eleva niveles de vulnerabilidad con respecto a la cultura. Es por ello fundamental tomar medidas preventivas para la protección del patrimonio arquitectónico de tal modo que el sentido de identidad no se pierda con el paso del tiempo.

- La reutilización como forma de adecuar de un mejor modo el patrimonio arquitectónico a las necesidades reales y contemporáneas de una comunidad, es una medida de beneficio mutuo en la que la comunidad se beneficia de la obra y esta última se beneficia de las actividades realizadas por la comunidad para darle nuevo uso. Es una forma efectiva de proteger al patrimonio arquitectónico, a la ciudad y al sentido de lugar.

- Cuando el patrimonio arquitectónico llega al momento de requerir reciclaje, significa que es necesario tomar medidas severas para poner al bien inmueble en un nuevo ciclo de vida. Esto incluye no solo la adaptación que se dio en la reutilización, sino, las labores de reparación, reposición, ajuste y si se quiere la re-construcción o ampliación de la obra que ha entrado en desuso. Evidentemente, el riesgo al historicismo existe, por lo que es importante, precisamente, el término de reciclaje ante las nuevas condiciones del sitio. El reto se encuentra en proteger el sentido de lugar a pesar de la introducción de elementos formales contemporáneos.

- Patrimonio, lugar y paisaje son compatibles con Khôra. La coexistencia de fondo y forma para entender el sentido de la existencia de una obra de arquitectura da coherencia a la relación entre la teoría y la práctica. Se propone incluir en los currículos de Arquitectura el estudio de Platón en el Timeo y de Derrida en el Khôra para enriquecer la estructuración cognitiva de los estudiantes.

- La relación entre arquitectura y antropología ofrece nuevas alternativas de investigación y de formación. Esta relación beneficia la protección al patrimonio arquitectónico. Visiones como las de Marc Augé señalan la importancia de superar las fronteras del conocimiento con el propósito de fortalecer las estructuras teóricas de la arquitectura. 


\section{Bibliografía}

ARAVENA, Alejandro. Las fuerzas en arquitectura: Alejandro Aravena Elemental. Revista ARQ. 2012. No 80, PP. 58-59.

ARISTOTELES, Física. Traducción y notas: Guillermo R. De Echandía Editorial Gredos S.A. 1995.

ASSELOT, Pierre. El paisaje del vino. Revista ARQ. 2003. No 54, PP. 56-61.

AUGÉ, Marc. Los "no lugares" espacios del anonimato: una antropología de la sobre-modernidad. Gedisa. 1996.

CRISPIANI, Alejandro. 2Un mundo continuo. Revista ARQ. 2001. No 49, PP. 57-59.

DERRIDA, Jaques. KHORA. Alción Editora. 1995.

DÍAZ, Francisco. Miedo a la arquitectura. Revista ARQ. 2011. No 77, PP. 13-14.

FELSENHARDT, Christina. Nuevas geometrías en viejos paisajes. Revista ARQ. 2003. No 54, PP. 10-14.

HOLMES, Jonathan. "Remodelación tienda UNICEF Chile. Revista ARQ. 2001. N. 49, PP. 47-47.
LECITRA, Micaela. Reducir, Reutilizar y Reciclar: El problema de los residuos sólidos urbanos. GEIC. 2010

MARTÍNEZ, Miguel. Reciclaje de arquitectura vs restauración arquitectónica, ¿herramientas contrapuestas?. Revista Hábitat y Sociedad, No 5 Noviembre 2012. P 23-33.

REUS, Patricia. Patrimonio arquitectónico y sostenibilidad: Reciclaje y participación, mecanismos para la revitalización urbana. Máster de Arquitectura y Urbanismo Sostenible. Universidad de Alicante. Julio 2012.

ROSAS, José. Territorializar la arquitectura. Revista ARQ. 2007. No 65, PP. 10-10.

SALDARRIAGA, Alberto. La arquitectura como experiencia: espacio, cuerpo y sensibilidad. Villegas Editores, Universidad Nacional de Colombia, 2002.

TEJEDA, Guillermo. "Diseño Patrimonial chileno". Revista ARQ. 2001. N. 49,Pp22-23

PLATON. Timeo. Edición de Patricio de Azcárate, Madrid 1872. http://www. greenpeace.org/mexico/es/Actua/ Ecotips/Las-tres-r/ 